\title{
Automatic segmentation of plantar thermograms using adaptive C means technique
}

\author{
Madhava Prabhu S. ${ }^{1}$, Seema Verma ${ }^{2}$ \\ ${ }^{1}$ Department of Electronics and Communication Engineering, Middle East College, Muscat, Sultanate of Oman \\ ${ }^{2}$ Department of Electronics, Banasthali University, Rajasthan, India
}

\begin{tabular}{l} 
Article Info \\
\hline Article history: \\
Received Aug 28, 2019 \\
Revised Aug 17, 2020 \\
Accepted Oct 9, 2020 \\
\hline
\end{tabular}

Keywords:

Adaptive

Diabetes

Image processing

Segmentation

Thermography

\begin{abstract}
Diabetic foot ulcer (DFU) is one of the major concern of diabetes and it is rapidly increasing, in worst case scenario this may lead to amputation. The DFU can be avoided by the early detection and proper diagnosis. Many of the studies carried out highlights that, thermography is the most useful technique to measure the changes in the temperature of plantar surface and alerts to indicate the risk associated with DFU. The distribution of temperature does not have a fixed pattern across the patients, hence it makes the difficulty in measuring the appropriate changes. This gap will provide a scope to improve the analysis technique so as to measure the plantar surface temperature effectively and identify any abnormal changes. In this paper, the segmentation algorithm namely adaptive $\mathrm{C}$ means (ACM) for the image segmentation is discussed. ACM is based on the spatial information and this method includes the two stage implementation. In the first stage, nonlocal spatial information is added and in the second stage, spatial shape information is used in order to refine the constraint of local spatial. Outcome of the proposed method shows that ACM is very much effective and it outperforms the other existing methods.
\end{abstract}

This is an open access article under the CC BY-SA license.

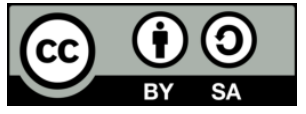

\section{Corresponding Author:}

Madhava Prabhu S.

Department of Electronics and Communication Engineering

Middle East College

Muscat, Sultanate of Oman

Email: madhav@mec.edu.om

\section{INTRODUCTION}

Diabetes is said to be one of the chronic diseases, which occurs when the human pancreas fails to produce the insulin. Insulin is the hormone that helps in regulating the blood sugar. Increase in the blood sugar level is the common effect observed in case of uncontrolled diabetes and this leads to the serious damage mainly to the blood vessels, nerves and kidneys [1]. Basically there are two type of diabetes i.e. type1 and type-2. The type-1 diabetes is non curable [2]. The common type of diabetes is type-2 that usually exists in adults. The recent data released by World Health Organization indicates that approximately 422 million people are living with diabetes worldwide and nearly 1.6 million deaths every year are directly related as cause of diabetes [3].

The common symptom is diabetic foot ulcer (DFU). DFU occurs when the reduced blood flow is combined along with the neuropathy (damage of nerve) in feet. DFU can be prevented, if it is detected in an initial stage. Currently this type of foot complication is identified by analyzing the foot neuropathy, plantar foot pressure and blood circulations. This approach provides the possibility to analyze the condition of patients suffering from foot ulcer [4]. However, these test normally causes the patients to visit the doctor 
more often and this is expensive as well as intrusive. Moreover the self-assessment is very much challenging since it all depends on the patient's knowledge regarding the disease [5].

Early detection of preulcerative lesions in the foot based on the several scenarios can prevent it from going to the hazardous stage [6]. For the patients at risk, the routine assessment of such foot complication is really beneficiary as there is no readily available instruments are available. This gap can be met by designing a thermal image processing based method to detect the potential DFUs. Thermography is nothing but the technique which is used for measuring the temperature distribution that occurs in tissues as well as organs [7]. This temperature distribution is namely known as thermogram. It is used in various conditions of planning the treatment of DFU [8]. Thermography is also capable of finding the region of interest (ROI). This technique is totally safe since it does not require physical interaction of humans and no introduction of instruments into the human body.

For thermogram analysis, there are mainly two approaches involved [9]. The characteristic patterns will be identified along with thermal variation measurement. Due to the fact that, the spatial patterns have wide variations across the diabetic patients [10], we proposed a methodology named as adaptive C-means (ACM), here both the information i.e. spatial shape and nonlocal spatial are utilized, and this helps in improvising thermogram analysis. In order to take benefit of nonlocal spatial information, weight on the objective function is added for increasing the pixel possibilities. Later on our method is compared with the existing segmentation methods. The comparison takes place in two ways i.e. quantitative as well as qualitative. The contralateral comparison of foot temperature technique [11] is not very much useful. When the temperature changes in both the feet and if both have no specific pattern, then one foot cannot used as a reference of the another. To overcome this, the proposed method tries to compare both the feet at a time. The proposed approach will improve the automatic classification and acts as an aid in assisting the clinical experts. Our research is organized in a way that second section describes the brief description about the existing work, whereas third section elaborates the proposed methodology, evaluation of our algorithm takes place in fourth section, this section also shows the case comparison of our proposed algorithm.

\section{LITERATURE REVIEW}

In this section, we present a brief review on the several segmentation approaches. The literature review shows that every technique has their advantages as well as shortcomings. This led us to design and develop our own methodology. There are limited computer methods developed for the assessment of diabetic foot pathologies.

Segmentation is a technique used for identifying the differences between particularly interesting and uninteresting parts. It is also used to differentiate the foreground from the background content. While comparing the several segmentation techniques [12], the thresholding technique was recommended. Though this method is simple to use, it was found that for certain images this technique fails show the clear difference between background content and feet [13]. The two different segmentation methods are compared by evaluating the performance on 10 samples each of diabetic and normal feet [14], the results highlighted that edge detection approach is consistently good in performance than the watershed method. The snake algorithm [15] is utilized to identify the ROI from the rest of the feet thermogram. It was also concluded that the snake algorithm is appropriate and relevant to plantar thermograms when compared with conventional segmentation techniques. The updated version with blended feature was introduced [16], and this technique showed better results than the traditional snake algorithm and also several segmentation techniques were evaluated to arrive at the decision.

A classification based on anatomy of foot was proposed [17], but this method is very tedious as the anatomy of individual's feet varies from patient to patient. This study is based on comparing the thermal distribution pattern of plantar region. Extracting the hottest region in plantar thermograms is very much essential to focus on relevant features. It can indicate some abnormalities. A simple method based on thresholding called as histogram-shape was used [18] to separate the hottest regions from the feet images. For plantar image segmentation, an active contour model was used, which was an extended version of thresholding to extract the hottest region [19]. In this study the ROI was divided into 6 portions to extract the relevant features.

One of the powerful approaches in the field segmentation technique is called as atlas-based. This approach needs expert knowledge in building the database which comprises information in the form a look up table (LUT) called as atlas and thus it is a limitation due to its complex structure [20]. The fuzzy $\mathrm{C}$ means (FCM) technique provides more accurate results than the $\mathrm{k}$ means and meanshift algorithms [21]. FCM is one of the unsupervised algorithm, which is a cluster based and not dependent on training and trained data. The one of the drawback with this approach is that, the traditional FCM technique only uses pixel membership information and does not make full use of spatial information around the pixel, so it is not ideal for noise 
reduction [22]. Some of the researchers have proposed several modified techniques for the FCM algorithm to improve the performance based on the intended application. One of the approach to improve the performance of FCM algorithm is making it self adaptive, where it can combine the neighborhood and non-neighborhood information of the image. The corresponding weight has to be calculated adaptively along with the addition of neighborhood spatial information to the clustering model [23]. The concept of markov random fields (MRF) is very much useful to make the FCM algorithm adaptive to the neighborhood systems [24]. The MRF is usually defined through a Gibbs function. The constraint of spatial context is addressed into the objective function of FCM to get a modified FCM algorithm.

When the literature review is analyzed, we observed that prior knowledge of shape information is very much essential as it helps in achieving the segmentation accuracy however there are some instances where it can fail when only shape information is considered. Hence to overcome these issues, a blended methodology which combines the advantage of traditional FCM along with MRF is addressed and it is discussed in the next section.

\section{PROPOSED METHODOLOGY}

In this section we present the proposed methodology i.e. adaptive C-means (ACM). This methodology contains two stages, first stage deals with including the non-local spatial information whereas the second stages includes the spatial information. The Figure 1 shows the flow of the proposed work. At first the parameters are initialized, parameter initialization is essential since it reduces the run time and this in turn helps in improvising the segmentation outcome. Later on the segmentation is done. Spatial and non-local spatial information based ACM is applied in order to achieve the better results. In segmentation there are mainly three steps. First step is initialization, here the parameters are initialized such as threshold parameter, fuzzy factor and number of clusters. In second stage the membership is updated, similarly in the third stage cluster centre (CC) are updated.

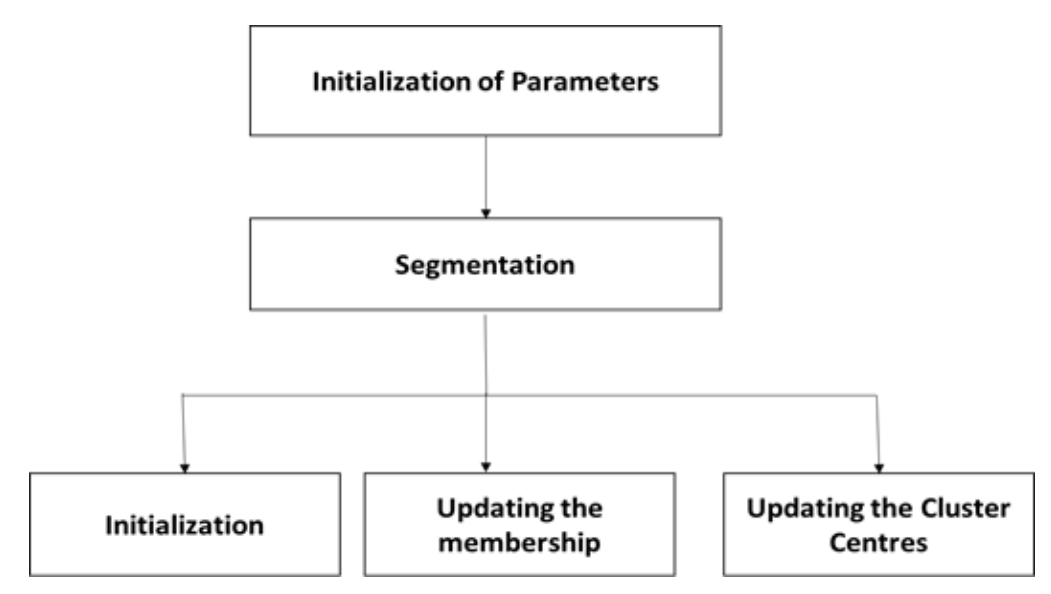

Figure 1. Flow of the proposed methodology

\subsection{ACM based on NSI (non-local spatial information)}

Here, the main intention is to reduce the error sum that exist between the centroid of each class and the intensity of given pixels. ACM technique reduces the error between each class's centroid and each pixel's intensity. The OF (object function) of FCM can be presented as:

$$
E_{F C M}=\sum_{i \in \Omega} \sum_{j=1}^{l} \mu_{i j}^{p}\left\|x_{i}-y_{j}\right\|^{2}
$$

where

$y_{j} \quad=$ Centroid

$\mu_{i j}=$ Membership

$x_{i}=$ Pixel's gray value at $i$ point

$\Omega \quad=$ Pixel location of the image

$p \quad=$ Segmentation value 
The membership function $\mu_{i j}$ satisfies the condition given as,

$$
\mu_{i j} \in[0,1], \sum_{j=1}^{l} \mu_{i j}=1
$$

As shown in (1), consideration in ACM that leads to being sensitive to the noise where spatial information is not present. To overcome this problem we use non-local SI (on basis of target image). In infrared (IR) images, usually the intensity is uneven [25]. Some pixels of the image might be incorrect while segmented by the algorithm, where centroid value is larger than the gray value in the image. To overcome this problem the pixel's weight might be constructed if the centroid and region of the target is known. If the pixel is closer to target's centroid, then it has high degree of the membership. The weight of each part is inversely proportional to the distance between centroid and pixel of the target approximately. To measure the distance, the Euclidean distance [26] method is used. To preserve the correct segment and masts details, the diagonal line's length of the target region is utilized for restraining the weight. The improved function with weighted term is shown below:

$$
\begin{aligned}
& E=\sum_{i \in \Omega} \sum_{j=1}^{l}\left(\mu_{i j} \eta_{i j}\right)^{p}\left\|x_{i}-y_{j}\right\|^{2} \\
& \eta_{i j}=\left(\frac{\mathfrak{D}}{\left(\left(b_{i}-b_{0}\right)^{2}+\left(a_{i}-a_{0}\right)^{2}\right)^{\frac{1}{2}}}\right)^{\frac{1}{j}}
\end{aligned}
$$

where,

$\eta_{i j} \quad=$ Weighted term

$\left(x_{i}, y_{i}\right)=$ Coordinate of pixel $i$

$\mathfrak{D} \quad=$ Diagonal line's length of possible region

$\left(x_{0}, y_{0}\right)=$ Coordinate of approx. centroid

The pixel $i$ is located at the possible region. If the clustering process has been executed at the possible region, so the value of $\frac{\mathcal{D}}{\left(\left(b_{i}-b_{0}\right)^{2}+\left(a_{i}-a_{0}\right)^{2}\right)^{\frac{1}{2}}}$ is greater than $1, \frac{1}{j}$ adjusts the weight of certain pixel's different class. For image class $c=3$, the value of weighted term will be cube root of distance weight. In case of class $c=1$, weighted term's value will be same as distance weight. Generally the value of $\frac{\mathfrak{D}}{\left(\left(b_{i}-b_{0}\right)^{2}+\left(a_{i}-a_{0}\right)^{2}\right)^{\frac{1}{2}}}$ is greater than 1 , so $\eta_{i 1}>\eta_{i 3}$. Then, the added weight might increase the memberships with respect to target image. The certain pixel's weight depending on different class has effective use and non-linear relationship with non-local spatial information. The value of $\left(x_{0}, y_{0}\right)$ and $\mathfrak{D}$ is fixed and possible region is predefined, that means during optimization process the value of $\eta_{i j}$ will be fixed.

\subsection{ACM based on SSI (spatial shape information)}

The MRF is defined by Gibb's distribution which is represented as prior probability,

$$
\begin{aligned}
& p_{s}(j)=e^{\frac{-F_{S}(j)}{Z}} \\
& Z=\sum_{j=1}^{l} e^{-F_{S}(j)}
\end{aligned}
$$

where,

$\mathrm{z}$ is the $\mathrm{NC}$ (Normalization constant).

$F_{S}(j)$ represents the energy that are mainly involved in the distribution of pixels.

The potential energy of a given pixel is described as,

$$
F_{s}(b)=\sum_{(s, t) \in C} G_{s, C\left(b \mid b_{t}\right)}
$$

In the above equation $b$ indicates the statistical indicant of given pixels whereas $b_{t}$ is statistical indicant of the pixel $t$, clique is denoted by $C$. When various clique $p . f$ (potential functions) $G_{s, C\left(b \mid b_{t}\right)}$ is selected, huge range of the distribution can be computed. Thus prior possibility of given pixel is formulated in the below equation.

$$
m_{s}(j)=\frac{1}{Z} e^{\left(-\sum_{w} \in N_{S}\left(1-\mu_{j, w}\right)\right)}
$$


$\mu_{j, w}$ is given members of pixel $r$, this belongs to the given class $j$, neighbors are indicated by $N_{s}$. Hence $\left(1-m_{s}(j)\right)$ indicates the refusable level, this shows that $N_{s}$ resistance has been used to assign the pixel to the label. After analyzing, refusable level is replaced by the following equation.

$$
\left(\left(1-m_{s}(j)\right) \times\left(1-p_{s}(j)\right)\right)^{1 / 2}
$$

Here, $\left(1-m_{s}(j)\right)$ does not change and the weight added is $\left(1-p_{s}(j)\right)$, it is applicable for revising the reusable levels. The energy involved in the distribution of pixels can be represented as,

$$
\begin{aligned}
& F_{s}(j)=\left(1-\mu_{j}(k-1, i-1)\right)\left(1+\mu_{j}(k+1, i+1)\right) \\
& +\left(1-\mu_{j}(k-1, i)\right)\left(1-\mu_{j}(k+1, i)\right) \\
& +\left(1-\mu_{j}(k, i-1)\right)\left(1-\mu_{j}(k, i+1)\right) \\
& +\left(1-\mu_{j}(k-1, i+1)\right)\left(1-\mu_{j}(k+1, i-1)\right)
\end{aligned}
$$

$\mu_{j}(k, i)$ is the member of the given pixel $(\mathrm{k}, \mathrm{i})$.

Moreover, local as well as nonlocal spatial information is utilized in order to improvise the FCM performance. Now, the OF (object function) of improvised FCM can be presented as,

$$
\begin{aligned}
& E_{A C M}=\sum_{i \in \Omega} \sum_{j=1}^{l}\left(\mu_{i j} \eta_{i j}\right)^{p}\left(\left(1-m_{s}(j)\right) \times\left(1-p_{s}(j)\right)\right)^{1 / 2} \\
& \times\left\|x_{i}-y_{j}\right\|^{2}
\end{aligned}
$$

In case of the constraint condition, to achieve the utmost object function value, a function is developed using the concept of Lagrange multipliers [27].

$$
\begin{aligned}
& f=\sum_{i \in \Omega} \sum_{j=1}^{l}\left(\mu_{i j} \eta_{i j}\right)^{p}\left(\left(1-m_{s}(j)\right) \times\left(1-p_{s}(j)\right)\right)^{1 / 2} \\
& \times\left\|x_{i}-y_{j}\right\|^{2}+\lambda\left(\sum_{j=1}^{l} \mu_{i j} \eta_{i j}-1\right)
\end{aligned}
$$

$\lambda$ is the Lagrange multiplier.

For simplifying the optimization issue, derivative of derived function is taken and it is presented in the (13). Membership function is formulated by the given equation.

$$
\mu_{i j}=\left(\frac{\frac{1}{\left\|x_{i}-y\right\|^{2}}\left(\frac{1}{\left(\left(1-m_{S}(j)\right) \times\left(1-p_{S}(j)\right)\right)}\right)}{\sum_{n=1}^{l} \frac{1}{\left\|x_{i}-y_{n}\right\|^{2}}\left(\frac{1}{\left(\left(1-m_{S}(j)\right) \times\left(1-p_{S}(j)\right)\right)}\right)}\right) / \eta_{i j}^{2}
$$

Each class has the centroid function that is presented in the (14).

$$
y_{j}=\frac{\sum_{i \in \Omega}\left(\mu_{i j}^{2} \eta_{i j}^{2}\left(\left(1-m_{s}(j)\right) \times\left(1-p_{s}(j)\right) \times x_{i}\right)^{1 / 2}\right)}{\sum_{i \in \Omega}\left(\mu_{i j}^{2} \eta_{i j}^{2}\left(\left(1-m_{s}(j)\right) \times\left(1-p_{s}(j)\right)\right)^{1 / 2}\right)}
$$

Once the nonlocal and local spatial information is introduced, ACM can easily outcast the issue which is caused by the intensity inhomogeneity, this clearly indicates that the proposed ACM methodology is novel as well as effective for the segmentation of plantar thermograms.

\section{RESULTS}

In this section we evaluate and present the results of the proposed algorithm. A total of 50 plantar thermograms were included in this study. The total number of diabetic group samples are 40 and remaining 
10 samples are from the controlled group. This algorithm worked perfectly for all the current images when compared with the ground result.

Generally, it will be possible to make an initial decision based on the visual inspection [28] to verify if the segmentation corresponding to an ROI was extracted correctly or not. In this inspection, it was found that the proposed algorithm, perfectly does the background-foreground segmentation. There were no cases of missing or additional features in the segmented image.

\subsection{Case comparison}

Under this category the results obtained from the proposed algorithm is compared with the snake algorithm. The snakes algorithm separates the feet from the background automatically. It can also be extended to separate the right foot from the left for each of the image [15]. The proposed technique makes use of adaptive method to extract the ROI from the background and it detects the foot contour better than the exisiting method. Later on, the extracted ROI can be used for further processing. The Table 1 provides the case comparision of the proposed technique with an existing technique.

Table 1. Comparison of existing technique with the proposed system

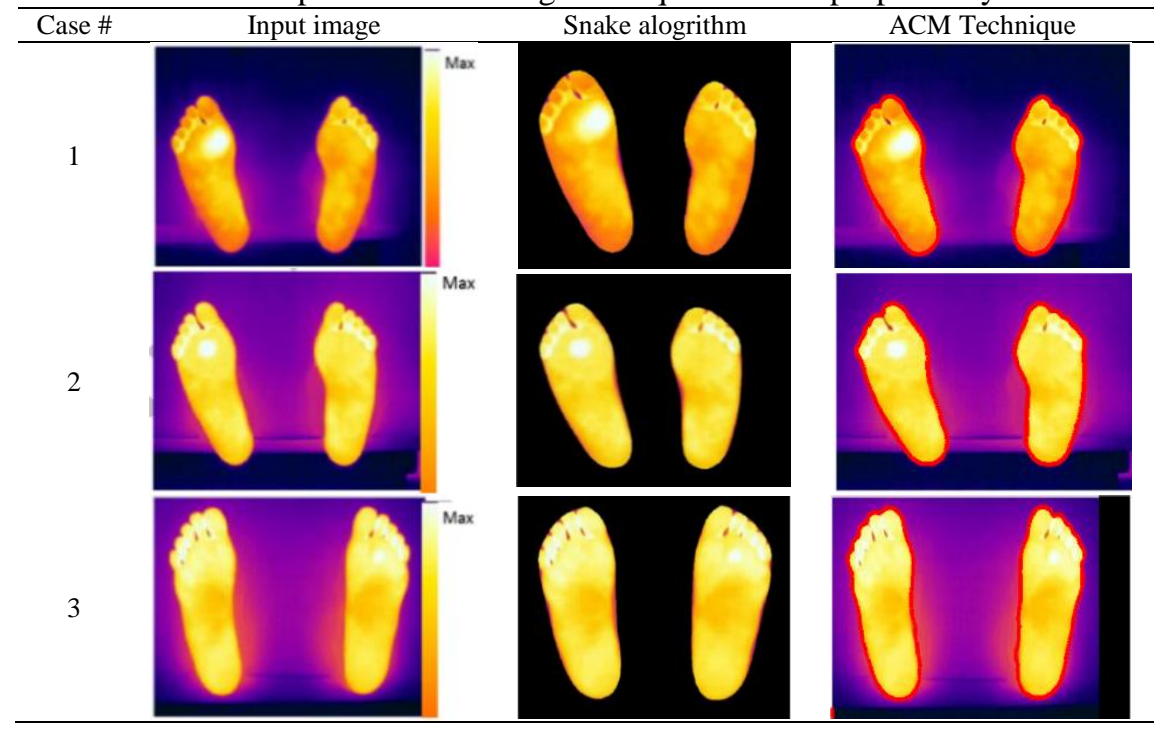

\subsection{Qualitative results}

In order to evaluate the proposed algorithm we have considered the several patient scenarios. Some of the selected cases out of 50 samples are listed in this section. The case numbers are identified using the nomenclature which is an easy way to detect the cases. The two letters are used to indicate the group. For control group, CG and for the diabetic group, DM is used as two letters code. Then it is followed by two digits corresponding to the serial numbers and the last letter indicates the gender of the subject. The letter $\mathrm{M}$ and $\mathrm{F}$ are used for representing male and $\mathrm{F}$ respectively. It is observed that in all the cases the proposed algorithm provides the closest contour to the ground results. The details of segmentation results obtained by the proposed method is provided in the Table 2 in reference to few of the cases tested.

\subsection{Performance metric evaluation}

In order to analyze the efficiency of a particular segmentation technique, there are several performance metric evaluation (PME) parameters are available. No formal way is followed to choose the metrics out of many for a particular segmentation task. The most of the researchers choose the evaluation metrics according to the popularity [29]. The selection of the best evaluation metrics depends on the segmentation goal based on the task to be achieved. These metrics will help in rating the various algorithms to select it properly and to achieve the intended performance [30]. The most appropriate two main PME parameters for plantar thermal image segmentation techniques are dice coefficient (DC) and root-meansquare deviation (RMSD). Let's use the notations $\mathrm{P}$ for the manually generated ground truth by the experts and Q for the result generated by the segmentation algorithm. The proposed work is compared with some of the existing works in the field of plantar thermal image segmentation techniques and the results are provided in the Table 3.

Automatic segmentation of plantar thermograms using adaptive C means technique (Madhava Prabhu S.) 
Table 2. Plantar thermograms of patients and segmentation results

\begin{tabular}{c|ccc}
\hline Case\# & Input image & ROI identified & Input with ROI superimposed \\
\hline DM20M & & \\
DM18M &
\end{tabular}

Dice coefficient, gives an idea about the similarity between two sets of data. It is also called as Sørensen-Dice index, the equation for measuring the DC is given by [31],

$$
D C=\frac{2 x|P| \cap|Q|}{|P|+|Q|}
$$

The cardinality of the set is represented by the vertical bars, i.e. the number of elements in that set, e.g. the number of elements in the set $\mathrm{A}$ is represented by $|\mathrm{A}|$. The elements that are common to both the sets are represented by the intersection $\cap$. The root-mean-square deviation (RMSD) is an indication of difference of the pixels throughout the images of ground truth and result generated by the segmentation algorithm. The equation for calculating the RMSD is given by [32],

$$
R M S D=\sqrt{\frac{1}{N} \sum_{i} \sum_{j}\left(P_{i j}-Q_{i j}\right)^{2}}
$$

Here, $\mathrm{N}$ is the size of the image.

The DC has the range between 0 (no overlap) to 1 (perfect match). The RMSD is always greater than 0 and if the value is 0 (which cannot be achieved in practice) indicates a perfect match between ground result and segmentation result. In general, higher value of DC and lower value of RMSD highlights better performance of the algorithm. The Table 3 gives an indication of better performance by the proposed method.

Table 3. PME score comparision

\begin{tabular}{ccc}
\hline Technique & DC & RMSD \\
\hline Snake [15] & 0.834 & 15.857 \\
Snake + Atlas [16] & 0.934 & 6.193 \\
Proposed method on ACM & 0.941 & 5.986 \\
\hline
\end{tabular}

\section{CONCLUSION}

In this paper, an effective as well as robust technique of segmentation is presented. The technique is named as adaptive $\mathrm{C}$ means (ACM). This approach is very efficient, less prone to noise and provides highly 
accurate results. Non-local spatial information (NSI) is applied by using the membership degree of each given pixel and spatial shape information (SSI) is helpful in minimizing the run time. When the result is compared with the existing similar model, it is observed that the proposed model provides the absolute ROI which helps in identifying the DFU. The approach can be extended and optimized for further processing. This approach is very much appropriate compared to conventional techniques such as thresholding, edge based and watershed based segmentations. The proposed method have shown some limitations as it an unsupervised algorithm. To detect the cold zones in the edges of toes, supervised algorithms can perform better as they are trained. Though this is just a step towards the detection of DFU, in future with the help of this image segmentation technique it can be implemented for feature extraction and it could help in upcoming research towards the better detection of the diabetes.

\section{ACKNOWLEDGEMENTS}

The authors would like to extend their special thanks to Mr. Shahul Hameed, Founder \& CEO, DIT, India for providing the necessary thermal images.

\section{REFERENCES}

[1] S. M. Prabhu and S. Verma, "Comparative Analysis of Segmentation techniques for Progressive Evaluation and Risk Identification of Diabetic Foot Ulcers," 2019 4th MEC International Conference on Big Data and Smart City (ICBDSC), Muscat, Oman, 2019, pp. 1-6.

[2] K. G. Osgouie and A. Azizi, "Optimizing fuzzy logic controller for diabetes type I by genetic algorithm," 2010 The 2nd International Conference on Computer and Automation Engineering (ICCAE), Singapore, 2010, pp. 4-8.

[3] WHO, "Diabetes," 2020. [Online]. Available at: https://www.who.int/health-topics/diabetes\#tab=tab_1.

[4] Ning Wang and Guixia Kang, "A monitoring system for type 2 diabetes mellitus," 2012 IEEE 14th International Conference on e-Health Networking, Applications and Services (Healthcom), Beijing, 2012, pp. 62-67.

[5] S. Rahaman, "Diabetes diagnosis decision support system based on symptoms, signs and risk factor using special computational algorithm by rule base," 2012 15th International Conference on Computer and Information Technology (ICCIT), Chittagong, 2012, pp. 65-71.

[6] M. Adam, E. Y. Ng, J. H. Tan, M. L. Heng, J. W. Tong, and U. R. Acharya, "Computer aided diagnosis of diabetic foot using infrared thermography: A review," Computers in Biology and Medicine, vol. 91, pp. 326-336, 2017.

[7] S. M. Prabhu and S. Verma, "A Systematic Literature Review for Early Detection of Type II Diabetes," 2019 5th International Conference on Advanced Computing \& Communication Systems (ICACCS), Coimbatore, India, 2019, pp. 220-224.

[8] T. Bernard, C. D'Elia, R. Kabadi and N. Wong, "An early detection system for foot ulceration in diabetic patients," 2009 IEEE 35th Annual Northeast Bioengineering Conference, Boston, MA, 2009, pp. 1-2.

[9] I. Cruz-Vega, D. Hernandez-Contreras, H. Peregrina-Barreto, J. Rangel-Magdaleno and J. Ramirez-Cortes, "Deep Learning Classification for Diabetic Foot Thermograms," Sensors, vol. 20, no. 6, p. 1762, 2020.

[10] Mori, T., Nagase, T., Takehara, K., Oe, M., Ohashi, Y., Amemiya, A., Noguchi, H., Ueki, K., Kadowaki, T., Sanada, H., "Morphological pattern classification system for plantar thermography of patients with diabetes," Journal of Diabetes Science and Technology, vol. 7, no. 5, pp. 1102-1112, 2013.

[11] Jones, B. F., "A reappraisal of the use of infrared thermal image analysis in medicine," IEEE Transactions on Medical Imaging, vol. 17, no. 6, pp. 1019-1027, 1998

[12] Kaabouch, N., Chen, Y., Hu, W.-C., Anderson, J. W., Ames, F., Paulson, R., "Enhancement of the asymmetry based overlapping analysis rough features extraction," Journal of Electronic Imaging, vol. 20, no. 1, p. 013012, 2011.

[13] Liu, C., van Netten, J. J., van Baal, J. G., Bus, S. A., van der Heijden, F., "Automatic detection of diabetic foot complications with infrared thermography by asymmetric analysis," Journal of Biomedical Optics, vol. 20, no. 2, p. 026003, 2015.

[14] G. L. Nandagopan and A. B. Haripriya, "Implementation and comparison of two image segmentation techniques on thermal foot images and detection of ulceration using asymmetry," 2016 International Conference on Communication and Signal Processing (ICCSP), Melmaruvathur, 2016, pp. 0356-0360.

[15] Etehadtavakol, M., Ng, E., Kaabouch, N., "Automatic segmentation of thermal images of diabetic-at-risk feet using the snake algorithm," Infrared Physics \& Technology, vol. 86, pp. 66-76, 2017.

[16] Asma, B., Harba, R., Canals, R., Ledee, R., Jabloun, R., "A joint snake and atlas-based segmentation of plantar foot thermal images," 2017 Seventh International Conference on Image Processing Theory, Tools and Applications (IPTA), Montreal, QC, Canada, 2017, pp. 1-6.

[17] T. Nagase, H. Sanada, K. Takehara, M. Oe, S. Iizaka, Y. Ohashi, M. Oba, T. Kadowaki, G. Nakagami, "Variations of plantar thermographic patterns in normal controls and non-ulcer diabetic patients: novel classification using angiosome concept," Journal of Plastic, Reconstructive and Aesthetic Surgery, vol. 64, no. 7, pp. 860-866, 2011.

[18] Hernandez-Contreras, D., Peregrina-Barreto, H., Rangel-Magdaleno, J., Ramirez-Cortes, J., Renero-Carrillo, F., "Automatic classification of thermal patterns in diabetic foot based on morphological pattern spectrum," Infrared Physics \& Technology, vol. 73, pp. 149-157, 2015. 
[19] Gururajarao, S. B., Venkatappa, U., Shivaram, J. M., Sikkandar, M. Y., Amoudi, A. A. "Infrared Thermography and Soft Computing for Diabetic Foot Assessment," Machine Learning in Bio-Signal Analysis and Diagnostic Imaging, pp. 73-97, 2019.

[20] Sharma N., and Aggarwal L. M., "Automated medical image segmentation techniques," Journal of Medical Physics, vol. 35, no. 1, pp. 3-14, 2010.

[21] M. Etehad Tavakol, S. Sadri, E. Y. K. Ng, “Application of K- and fuzzy c-Means for color segmentation of thermal infrared breast images," Journal of Medical Systems, vol. 34, no. 1, pp. 35-42, 2010.

[22] K. Chuang, "Fuzzy c-means clustering with spatial information for image segmentation," Computerized Medical Imaging and Graphics, vol. 30, no. 1, pp. 9-15, 2006.

[23] M. Li, "Adaptive Segmentation of Remote Sensing Images Based on Global Spatial Information," Sensors (Basel), vol. 19, no. 10, p. 2385, 2019.

[24] Y. Hong Lei, "Remote Sensing Classification Using Fuzzy C-means Clustering with Spatial Constraints Based on Markov Random Field," European Journal of Remote Sensing, vol. 46, no. 1, pp. 305-316, 2013.

[25] L. Vilcahuaman, et al., "Detection of diabetic foot hyperthermia by infrared imaging," 2014 36th Annual International Conference of the IEEE Engineering in Medicine and Biology Society, Chicago, IL, 2014, pp. 4831-4834.

[26] S. Purnima, "Automated Detection of Diabetic Foot Using Thermal Images by Neural Network Classifiers," International journal of Emerging Trends in Science and Technology, vol. 04, no. 05, pp. 5183-5188, 2017.

[27] V. Vela-Rincón, "Spatial alpha-Trimmed Fuzzy C-Means Algorithm to Image Segmentation," Lecture Notes in Computer Science, pp. 118-128, 2020.

[28] J. Gauci, "Automated Region Extraction from Thermal Images for Peripheral Vascular Disease Monitoring," Journal of Healthcare Engineering, vol. 2018, pp. 1-14, 2018.

[29] A. A. Taha, A. Hanbury and O. A. J. Del Toro, "A formal method for selecting evaluation metrics for image segmentation," 2014 IEEE International Conference on Image Processing (ICIP), Paris, 2014, pp. 932-936.

[30] S. Kumar, "Performance Metric Evaluation of Segmentation Algorithms for Gold Standard Medical Images," Recent Findings in Intelligent Computing Techniques, vol. 709, pp. 457-469, 2018.

[31] C. Moore, et al., "Dice similarity coefficient|Radiology Reference Article|Radiopaedia.org," Radiopaedia.org, 2020. [Online]. Available: https://radiopaedia.org/articles/dice-similarity-coefficient.

[32] S. Glen, "RMSE: Root Mean Square Error-Statistics How To," Statistics How To, 2020. [Online]. Available: https://www.statisticshowto.com/probability-and-statistics/regression-analysis/rmse-root-mean-squareerror/\#: :text=Root\%20Mean\%20Square\%20Error\%20(RMSE)\%20is\%20the\%20standard\%20deviation\%20of,the $\% 20$ line\%20of\%20best\%20fit.

\section{BIOGRAPHIES OF AUTHORS}

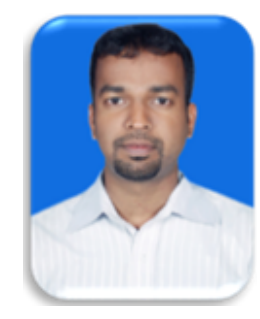

Madhava Prabhu S. obtained his Master degree in Embedded Systems from Manipal University India, and Bachelor degree in Electronics \& Communication Engineering from Visvesvaraya Technological University India in 2008 and 2005 respectively. His research interest are singal processing and embedded systems.

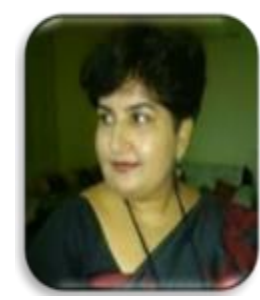

Seema Verma obtained her M.Tech and Ph.D degree from Banasthali University in 2003 and 2006 respectively. She is working as a Professor in the department of Electronics. Her research areas are VLSI Design, communication Networks and signal processing. 